\title{
Design and Analysis of a Broadband Microwave Amplifier
}

\author{
Obinna Okoyeigbo ${ }^{1}$, Augustus E. Ibhaze ${ }^{2}$, Ayobami Olajube ${ }^{3}$, Olamilekan Shobayo ${ }^{4}$, \\ Tobiloba Somefun ${ }^{5}$, Onyinyechi Steve-Essi ${ }^{6}$ \\ 1,3,4,5,6 Department of Electrical and Information Engineering, Covenant University, Ota, Ogun State, Nigeria \\ ${ }^{2}$ Department of Electrical and Electronic Engineering, University of Lagos, Akoka, Lagos State, Nigeria
}

\begin{tabular}{l} 
Article Info \\
\hline Article history: \\
Received Sep 8, 2020 \\
Revised Nov 27, 2020 \\
Accepted Dec 17, 2020 \\
\hline Keywords: \\
Broadband \\
Microwave Amplifier \\
Microstrip \\
Agilent \\
Advanced Design System (ADS)
\end{tabular}

\begin{abstract}
This paper presents the procedures involved in the design and analysis of a microstrip broadband microwave amplifier. For system design, simulation, optimization and analysis, a Computer Aided Design (CAD) tool known as Agilent Advanced Design System (ADS) was employed. The amplifier device- FLC317MG-4 FET, was tested for stability, and was observed to be unconditionally stable between 2 to $6 \mathrm{GHz}$ frequency band. Two possible ideal matching circuits were investigated to identify the best matching circuit with the maximum transducer power gain. It was observed that the quarter-wave transformer with parallel open circuit stub, gave a high gain at a wider range of frequency (larger bandwidth/ broadband), than the other matching circuit. Hence, it was employed for the broadband amplifier design using microstrips, and achieved a maximum flat gain of about $9.8 \mathrm{~dB}$ to $10.118 \mathrm{~dB}$, at a bandwidth of 3.5 to $4.5 \mathrm{GHz}$.
\end{abstract}

Copyright $\odot 2020$ Institute of Advanced Engineering and Science. All rights reserved.

\section{Corresponding Author:}

Obinna Okoyeigbo,

Department of Electrical and Information Engineering,

Covenant University,

Ota, Ogun State, Nigeria.

Email: obinna.okoyeigbo@covenantuniversity.edu.ng

\section{INTRODUCTION}

The evolution of wireless communication has provided numerous applications for RF and microwave amplifiers. RF/microwave amplifiers are vital components employed in various electronic and communication system applications, including base station equipment, wireless telephones, satellites, radar applications, magnetic resonance imaging (MRI), broadcasting, global positioning system (GPS), air traffic systems etc [1].

Amplifiers are devices that increase the output power of signals. Amplifies are used whenever a weak signal is received, and needs to be boosted. Microwave amplifiers amplify at microwave frequencies. Various categories of RF/ microwave amplifiers exists. They include broadband, narrowband, variable gain, buffer, low-noise, and high-efficiency amplifiers etc. Broadband amplifiers amplify over a wide range of frequency, without significant losses within the passband. Broadband amplifiers, which have good matching properties, high power output, broad/wide bandwidth and low nonlinear distortion, are among the most widely used amplifier type in wireless communications [2]. The wide bandwidth enhances the data rate, while the increased power output increases the distance of communication. Thereby satisfying the requirements of modern communication networks. Broadband amplifiers provide a number of other advantages. They do not require resonant circuit tuning, and it is possible to transmit a wide multimode signal spectrum or to achieve fast frequency agility [3].

Computer Aided Design (CAD) tools are extensively used in the design of RF/microwave amplifiers and various engineering applications, for better systems optimization and analysis, to accelerate the design process, and reduce cost of producing numerous prototypes before the final implementation stage $[4,5,6]$. RF/ microwave amplifier design, basically requires CAD tools [7, 8], for stability analysis, device modeling, Sparameters, matching and biasing networks etc. 
This paper looks at the design of a microstrip broadband amplifier, at a microwave frequency range of 3.5 to $4.5 \mathrm{GHz}$, using Agilent ADS (Advance Design System).

\section{THEORETICAL BACKGROUND}

In amplifier design, it is necessary to ensure maximum gain, stability and minimal losses. All these steps are discussed, and some terms and parameters that are required for a proper understanding of amplifier design process are also discussed.

\subsection{Gain}

This refers to the ratio of the magnitude of the power output to the magnitude of the power input. Three types of amplifier gain definitions exists. They are:

- Power Gain $\left(\mathrm{G}_{\mathrm{P}}\right)$ : this is the ratio of the power supplied to the load, to the power supplied to the amplifier.

- Available Gain $\left(\mathrm{G}_{\mathrm{A}}\right)$ : this is the ratio of the amplifier power output to the available source power.

- Transducer Gain $\left(\mathrm{G}_{\mathrm{T}}\right)$ : this is the ratio of the power supplied to the load, to the available source power. In amplifier design, the transducer gain is the most important parameter in determining its effectiveness and performance. The transducer gain is given as:

$$
G_{T}=\frac{1-\left|\Gamma_{S}\right|^{2}}{\left|1-S_{11} \Gamma_{S}\right|^{2}}\left|S_{21}\right|^{2} \frac{1-\left|\Gamma_{L}\right|^{2}}{\left|1-S_{22} \Gamma_{L}\right|^{2}}
$$

Where $\Gamma_{S}$ and $\Gamma_{L}$ are the source and load reflection coefficient respectively, $S_{11}$ and $S_{22}$ are the reflection coefficient at port 1 and 2 respectively, $S_{21}$ is the forward transfer gain, and $S_{12}$ is the reverse gain.

\subsection{Maximum Gain}

The maximum (unilateral when $\mathrm{S}_{12}=0$ ) gain is the best possible gain that can be achieved. It is the optimum gain of the amplifier, and can be achieved when the input and output networks of the amplifier are conjugate matched [9] to the transistor, and when the system is stable. It is determined by the S-parameters, i.e when $\Gamma_{\mathrm{S}}=\mathrm{S}_{11}$ *, and $\Gamma_{\mathrm{L}}=\mathrm{S}_{22} *$. Maximum Gain is given as [10]:

$$
G_{T U_{M A X}}=\frac{\left|S_{21}\right|^{2}}{\left.|1-| S_{11}\right|^{2}|| 1-\left|S_{22}\right|^{2} \mid}
$$

An important factor to consider in amplifier design is the trade-off between the gain and bandwidth. This is shown by the transfer characteristics of an amplifier with lumped coupling elements [11]. Ref. [12] discusses how this trade-off can be avoided, while [13] proposes how the bandwidth accuracy and bandwidth gain-independence can be increased.

\subsection{Stability Analysis}

Device stability is indeed an important factor to consider in amplifier design [14]. This is because an amplifier which is unstable may act as an oscillator, which is undesirable [10]. Hence, it is necessary to investigate if the active device is conditionally stable or unconditionally stable $[15,16,17]$.

An amplifier is unconditionally stable if $\left|\Gamma_{S}\right|<1$, and $\left|\Gamma_{\mathrm{L}}\right|<1$, for all passive source and load impedances, and conditionally stable only for a certain range of passive source and load impedances [16]. The stability of an amplifier is frequency dependent. An amplifier can be stable at a particular frequency, but unstable at another. A trade-off exists between the stability and bandwidth of an amplifier [18].

According to Rollett's stability criteria [10, 15, 19], an amplifier is assumed to be unconditionally stable if:

$$
K=\frac{1-\left|S_{11}\right|^{2}-\left|S_{22}\right|^{2}+|\Delta|^{2}}{2\left|S_{12} S_{21}\right|}>1
$$

and

$$
b=1+\left|S_{11}\right|^{2}-\left|S_{22}\right|^{2}-|\Delta|^{2}>0
$$


Where K represents Rollett's Stability Factor, $\Delta=S_{11} S_{22}-S_{12} S_{21}$, and b represents the Stability Measure.

\subsection{Input and Output Matching Circuits}

In other to maximize the gain, for maximum power transfer, and to minimize losses due to reflections, matching of both the input and output network is required [9]. In the design of an amplifier, maximum gain can be achieved by tuning the circuit components with the tuning function in ADS, or by matching the input and output circuits using a smith chart.

\section{DESIGN PROCEDURE AND RESULTS}

The process of amplifier design includes some important steps, which must be followed carefully, to achieve the desired result. These procedures and key parameters are summarized as follows:

\subsection{Stability Analysis}

In amplifier design, it is necessary as a first step, to investigate the stability of the active device which will be used [10, 15]. This is because, it is one of the most important characteristics of an amplifier, (else it becomes an oscillator).

The stability of the device- FLC317MG-4 FET, with a drain source voltage of $10 \mathrm{~V}$ and drain current of $720 \mathrm{~mA}$, as shown in Figure 1 was tested using ADS.

Other key parameters include:

- $\quad$ Frequency Sweep range of $2 \mathrm{GHz}-6 \mathrm{GHz}$ and steps of $0.5 \mathrm{GHz}$.

- $\quad$ Centre Frequency of $4 \mathrm{GHz}$.

- $\quad$ Load and Source terminals of $50 \mathrm{Ohm}$ each.

The circuit was simulated, and the S-parameters, the stability factor $(K)$ and the stability measure (b), of the transistor device was gotten.

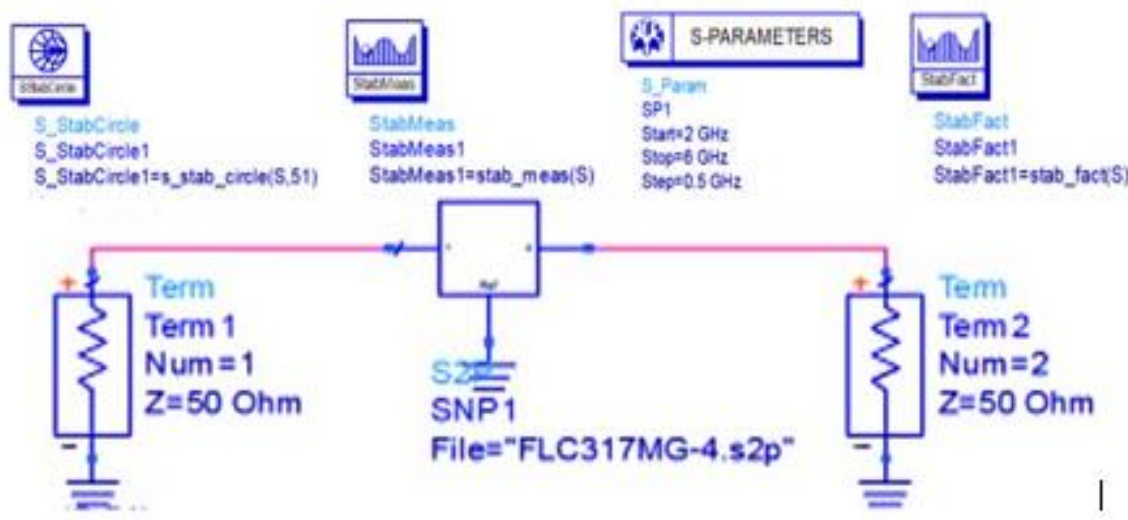

Figure 1. Circuit representation for the FLC317MG-4 FET Device Stability Analysis.

Results: After simulation of the FET device in Figure 1, the results of the stability analysis was given in Table 1 and Figure 2.

Table 1. S-Parameters of the FLC317MG-4 FET Device.

\begin{tabular}{rcccc}
\hline Frequency & \multicolumn{1}{c}{$\mathbf{S}(\mathbf{1 , 1})$} & $\mathbf{S}(\mathbf{1}, \mathbf{2})$ & $\mathbf{S}(\mathbf{2 , 1})$ & $\mathbf{S}(\mathbf{2 , 2})$ \\
\hline $2.0 \mathrm{GHz}$ & $-0.773+\mathrm{j} 0.459$ & $0.013+\mathrm{j} 0.019$ & $0.602+\mathrm{j} 2.018$ & $-0.520-\mathrm{j} 0.049$ \\
$2.5 \mathrm{GHz}$ & $-0.641+\mathrm{j} 0.610$ & $0.012+\mathrm{j} 0.024$ & $0.736+\mathrm{j} 1.750$ & $-0.554-\mathrm{j} 0.027$ \\
$3.0 \mathrm{GHz}$ & $-0.438+\mathrm{j} 0.732$ & $0.017+\mathrm{j} 0.026$ & $0.891+\mathrm{j} 1.537$ & $-0.588+\mathrm{j} 0.007$ \\
$3.5 \mathrm{GHz}$ & $-0.151+\mathrm{j} 0.785$ & $0.015+\mathrm{j} 0.032$ & $1.323+\mathrm{j} 1.500$ & $-0.612+\mathrm{j} 0.054$ \\
$4.0 \mathrm{GHz}$ & $0.184+\mathrm{j} 0.650$ & $0.028+\mathrm{j} 0.032$ & $1.820+\mathrm{j} 0.996$ & $-0.639+\mathrm{j} 0.106$ \\
$4.5 \mathrm{GHz}$ & $0.318+\mathrm{j} 0.189$ & $0.045+\mathrm{j} 0.017$ & $2.530+\mathrm{j} 0.270$ & $-0.687+\mathrm{j} 0.213$ \\
$5.0 \mathrm{GHz}$ & $-0.276+\mathrm{j} 0.006$ & $0.032-\mathrm{j} 0.022$ & $1.789-\mathrm{j} 1.605$ & $-0.678+\mathrm{j} 0.430$ \\
$5.5 \mathrm{GHz}$ & $-0.388+\mathrm{j} 0.629$ & $-0.007-\mathrm{j} 0.021$ & $0.154-\mathrm{j} 1.512$ & $-0.513+\mathrm{j} 0.614$ \\
$6.0 \mathrm{GHz}$ & $0.012+\mathrm{j} 0.885$ & $-0.020-\mathrm{j} 0.006$ & $-0.179-\mathrm{j} 0.725$ & $-0.340+\mathrm{j} 0.707$ \\
\hline
\end{tabular}




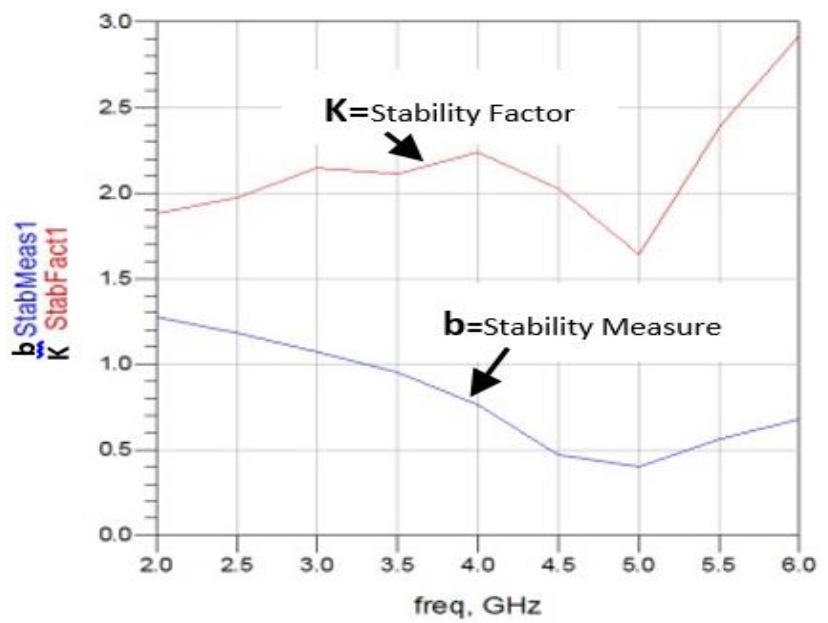

Figure 2. Stability Factor and Stability Measure of the FLC317MG-4 FET Device.

\section{Comments:}

A device is assumed to be stable if $S_{11}<1$ and $S_{22}<1$. According to the $S$ parameters, it was observed that the device is stable at the frequency range between $2-6 \mathrm{GHz}$.

According to the stability analysis, an amplifier is said to be unconditionally stable if the stability measure $(b)$ is greater than zero, and the stability factor $(K)$ is greater than 1 . According to the stability measure and stability factor plots shown in Figure 2, the device was observed to be unconditionally stable within the bandwidth of 2-6 GHz.

It was also observed that the forward transfer gain $S_{21}$ is much greater than $S_{12}$, which further confirms the stability of the system, and proper matching of the device. Hence, the device is potentially stable at the given frequency range 2-6 GHz, but could be potentially unstable at other frequency ranges.

\subsection{Ideal Matching Circuit}

In other to match the circuit, and get the maximum transducer gain, the values for the Simultaneous Match-Input Impedance (SmZ1) and Simultaneous Match-Output Impedance (SmZ2) were generated at 4GHz (centre frequency) using the SmZ1 and SmZ2 functions in the ADS and given as: SmZ1 = $32.264-\mathrm{j} 57.757 \Omega$, and $\mathrm{SmZ2}=14.160-\mathrm{j} 4.85 \Omega$.

The SmZ1 and SmZ2 give an output value of zero when the device is unstable, and tells us the conjugate matching impedances when the device is unconditionally stable. The values of SmZ1 and SmZ2 at $4 \mathrm{GHz}$ were used to determine the best matching network (i.e the network with the highest gain and widest bandwidth) out of two possible matching networks, which are:

- Short circuit parallel stub with series transmission line,

- Quarter-wave transformer with parallel open circuit stub. as follows:

Using the Smith Chart, with the values of SmZ1 and SmZ2 generated, the two networks were matched

\subsubsection{Short Circuit Parallel Stub with Series Transmission Line}

In the case of the input matching, the source impedance was set to be $\mathrm{SmZ1}$, which was connected in series to the transmission line, and the transmission line was connected to a $50 \Omega$ load impedance in parallel with a short circuit parallel stub. The circuit was matched (from source to load) by setting the impedance values

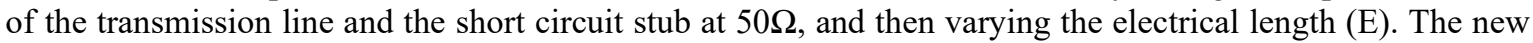
matched values of the electrical length for the input and output were recorded in Table 2.

For the output matching, the same procedure was performed as the input, but changing the source impedance to SmZ2.

Table 2. Matched Circuit parameters for the Short circuit parallel stub with series transmission line.

\begin{tabular}{lrrrrr}
\hline & \multicolumn{2}{c}{ Parallel Short Circuit Stub } & \multicolumn{2}{c}{ Transmission Line } \\
\cline { 2 - 6 } & $\mathrm{Z}(\mathrm{Ohm})$ & & $\mathrm{E}(\mathrm{Deg})$ & $\mathrm{Z}(\mathrm{Ohm})$ & $\mathrm{E}(\mathrm{Deg})$ \\
\hline Input Matching $(\mathrm{SmZ1})$ & 50 & 33.7 & 50 & 36.392 \\
Output Matching (SmZ2) & & 50 & 100.035 & 50 & 146.464 \\
\hline
\end{tabular}




\subsubsection{Quarter-wave Transformer with Parallel Open Circuit Stub}

For the input matching, the source impedance was set to be $\mathrm{SmZ1}$, which was connected in series to a quarter-wave transformer with a fixed length of $90^{\circ}$, and then matched to the $50 \Omega$ load impedance through a parallel open circuit stub.

For the output matching, the same procedure was performed as the input, but changing the source impedance to SmZ2. The new matched values of the electrical length (E) and impedance $(\mathrm{Z})$ were recorded in Table 3.

Table 3. Matched Circuit parameters for Quarter-wave transformer with parallel open circuit stub.

\begin{tabular}{|c|c|c|c|c|}
\hline & \multicolumn{2}{|c|}{ Quarter-wave Transformer } & \multicolumn{2}{|c|}{ Parallel Open Circuit Stub } \\
\hline & $\mathrm{Z}(\mathrm{Ohm})$ & $\mathrm{E}($ Deg) & $\mathrm{Z}(\mathrm{Ohm})$ & E(Deg) \\
\hline (SmZ1) & 83.2 & 90 & 50 & 33.3 \\
\hline (SmZ2) & 28 & 90 & 50 & 46.6 \\
\hline
\end{tabular}

\subsection{Ideal Maximum Transducer Power Gain}

After the two circuits have been matched using smith chart, the values of the various impedance and electrical lengths were used to design the two input and output circuit components of the amplifier. The two circuits were simulated, and the Maximum Gain, and S-parameter plots were generated and analysed for both circuits.

\subsubsection{Short Circuit Parallel Stub with Series Transmission Line}

The amplifier circuit shown in Figure 3 was designed according to the matched circuit parameters given in Table 2.

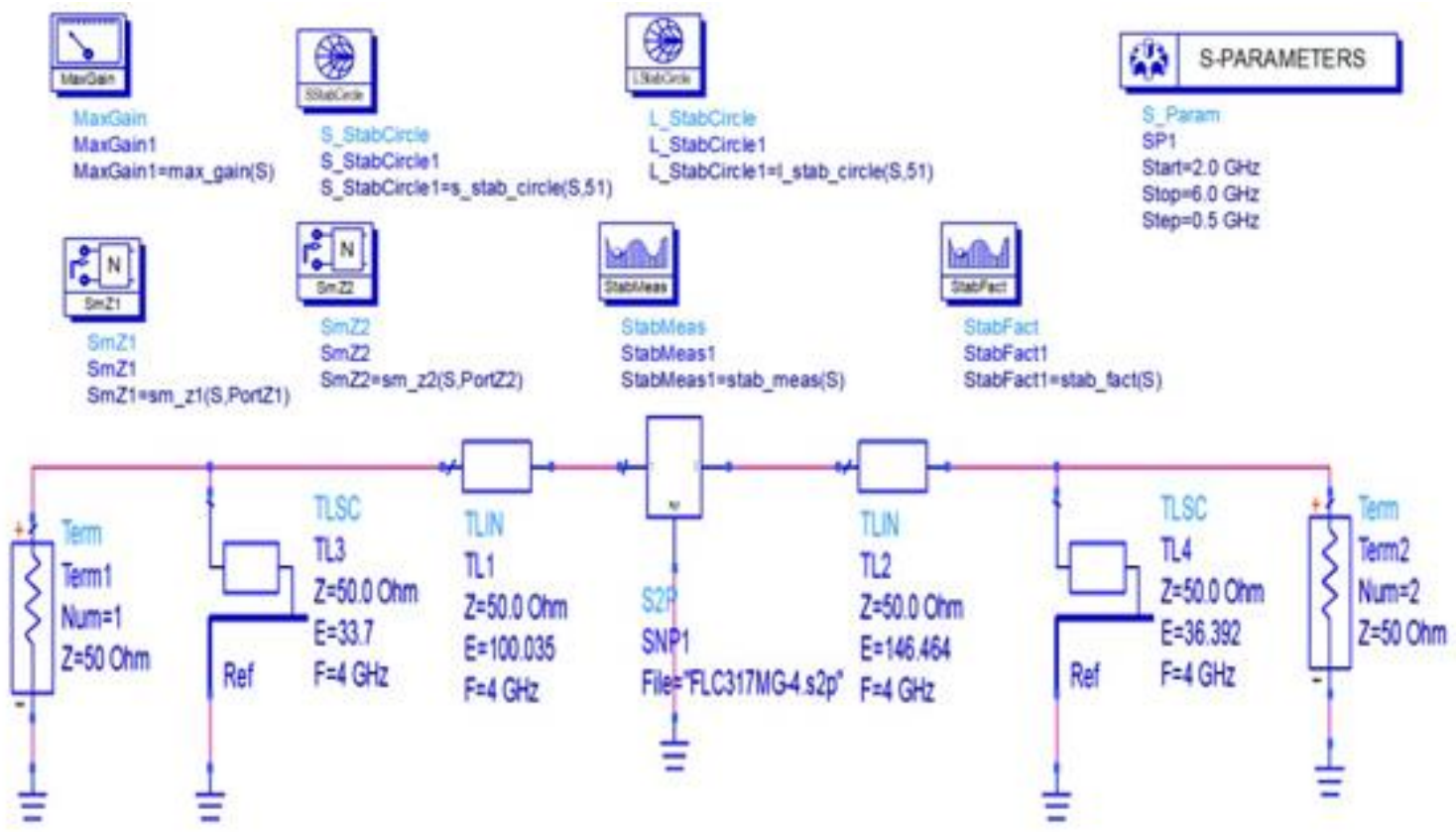

Figure 3. Circuit Schematic of the Short circuit parallel stub with series transmission line.

Results: The amplifier circuit shown in Figure 3 was simulated, and the maximum gain response and return loss obtained is shown in Figure 4. 


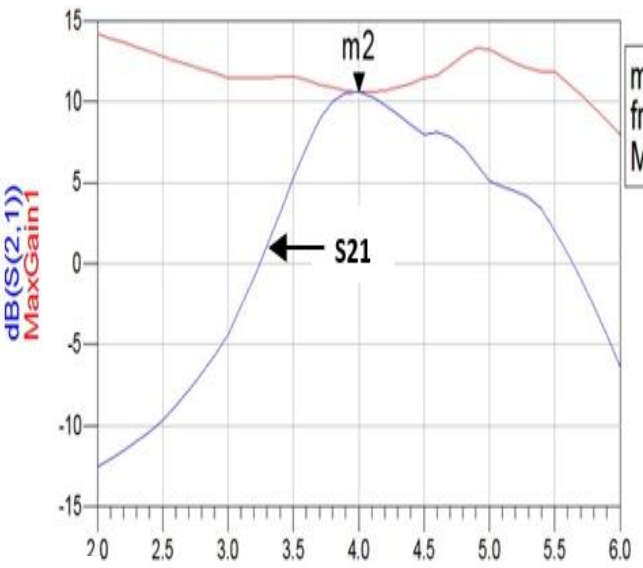

(a)

freq, GHz

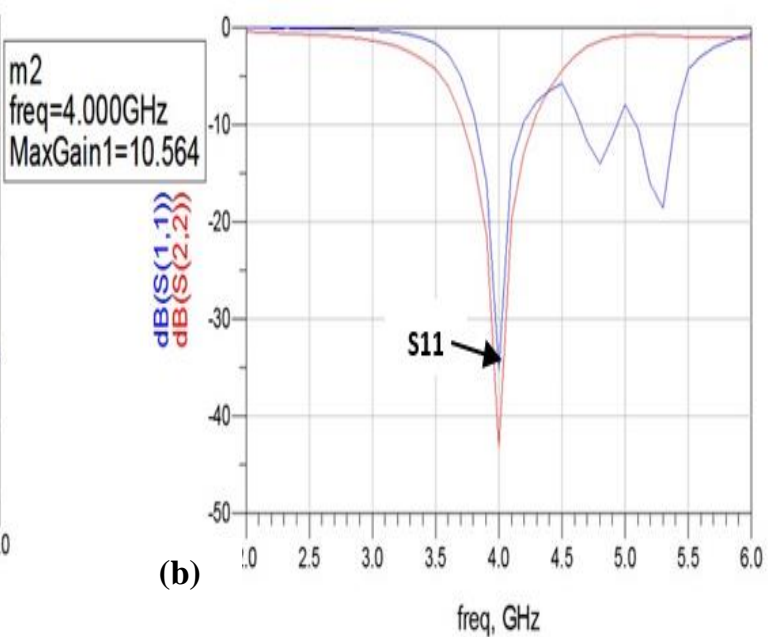

Figure 4. (a) Ideal Maximum Transducer Power Gain $\mathrm{S}_{21}$ and (b) Reflection Coeffcient $\mathrm{S}_{11}$ for Short circuit parallel stub with series transmission line.

Comments: From Figure 4, the maximum gain of the amplifier was observed to be $10.564 \mathrm{~dB}$, which represents the maximum transfer gain $\mathrm{S}_{21}$, (showing that the circuit is matched) and occurs at frequency of $4 \mathrm{GHz}$. It was also observed that at $4 \mathrm{GHz}$, the return loss was very low. This could imply that at $4 \mathrm{GHz}$, the amplifier would yield maximum amplification with little loss or reflection.

It was also observed that the gain is high at a very small range of frequency (i.e narrowband). It was also observed that $\mathrm{SmZ1}$ and $\mathrm{SmZ2}$ had values of about $50 \Omega$ at $4 \mathrm{GHz}$, which shows that the input and output circuit is matched.

\subsubsection{Quarter-wave Transformer with Parallel Open Circuit Stub} Table 3.

The amplifier circuit in Figure 5 was designed according to the matched circuit parameters given in

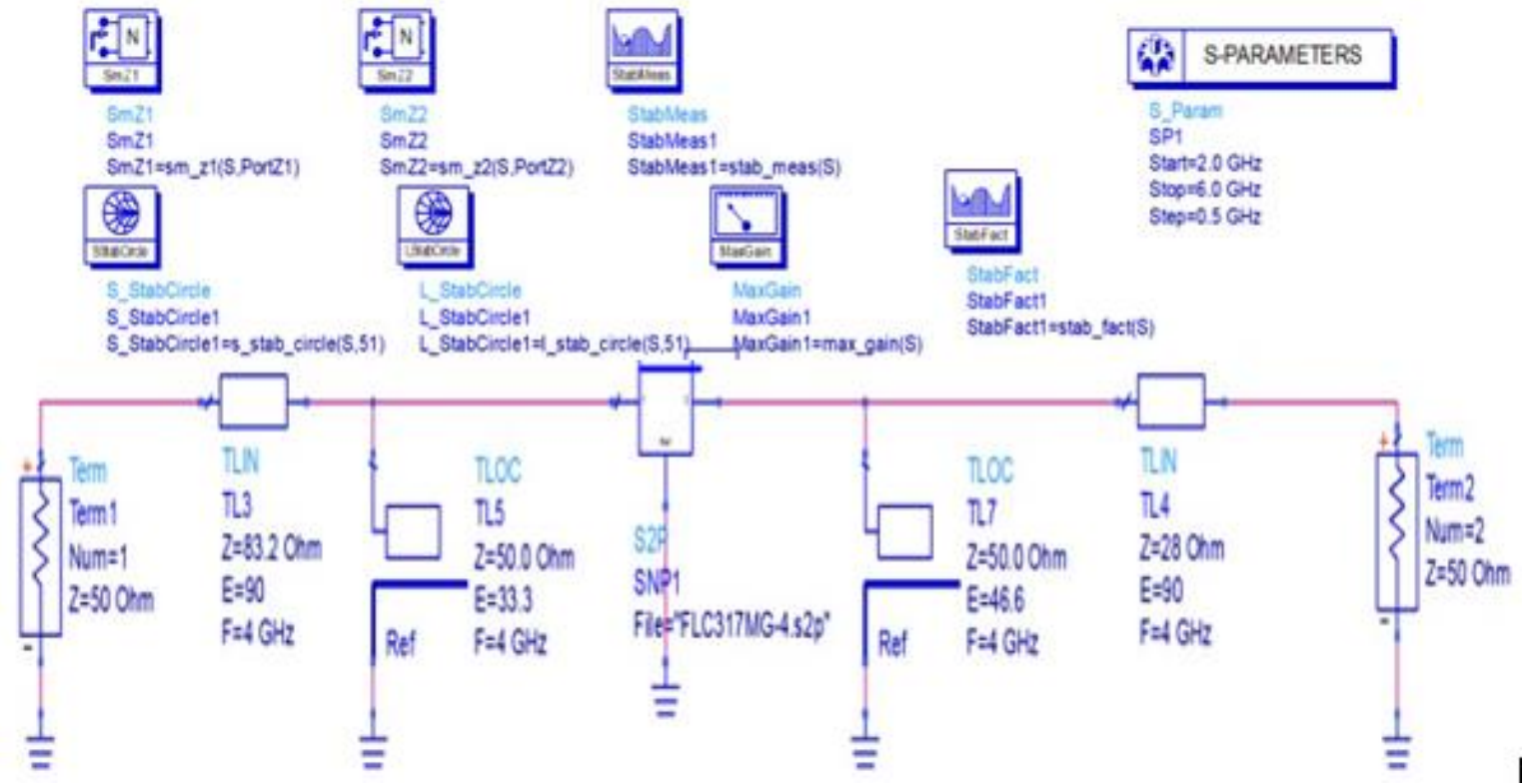

Figure 5. Circuit Schematic of the Quarter-wave transformer with parallel open circuit stub.

Results: The amplifier circuit in Figure 5 was simulated, and the maximum gain response and reflection coeffcient obtained is shown in Figure 6. 

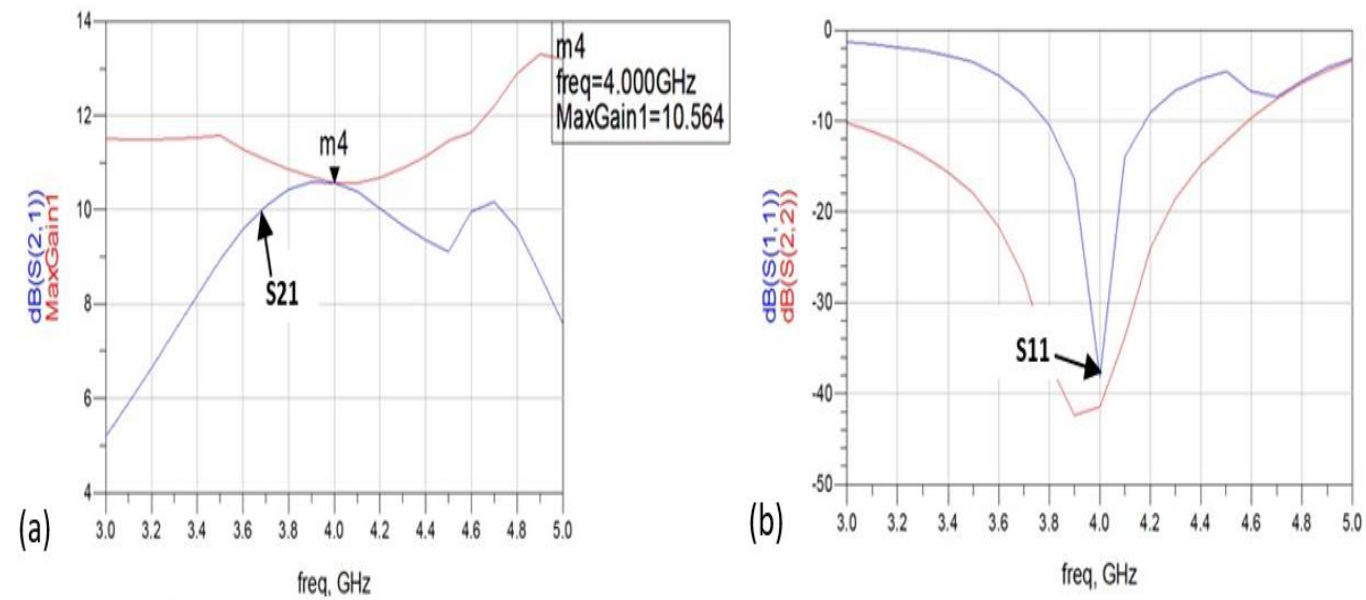

Figure 6. (a) Ideal Maximum Transducer Power Gain $\mathrm{S}_{21}$ and (b) Reflection Coeffcient $\mathrm{S}_{11}$ for Quarter-wave transformer with parallel open circuit stub.

Comments: From Figure 6, It was shown that the maximum gain of the amplifier is 10.564dB, (in its ideal case) which represents the maximum transfer gain $\mathrm{S}_{21}$, showing that the circuit is matched and occurs at $4 \mathrm{GHz}$. It was also observed that $\mathrm{SmZ} 1$ and $\mathrm{SmZ} 2$ had values of about $50 \Omega$ at $4 \mathrm{GHz}$, which shows that both the input and output circuit is matched.

It was also observed that for both matching circuits, we achieved same high gain, but the quarter-wave transformer with parallel open circuit stub, gave a higher gain at a very wide range of frequency (larger bandwidth/ broadband). In other words, the quarter-wave transformer with parallel open circuit stub, gave a flat gain at a wider range of frequency than the short circuit parallel stub with series transmission line. As a result, this was considered to be the best match broadband amplifier circuit, which shall be employed using the microstrip.

\subsection{Microstrip Broadband Amplifier with Constant Transducer Power Gain}

For a more practical design, the MLIN microstrip physical line models are employed to replace the ideal lines. The amplifier specification is a constant $10+/-0.2 \mathrm{~dB}$ flat gain, with a bandwidth of 3.5 to $4.5 \mathrm{GHz}$. Using the gain circle tool under Simulation S_Param palette in the ADS, the locus of reflection coefficients that would yield the maximum gain, was seen on the smith chart, and the conjugate matching impedances required for both the input (and output) matching networks were determined. The gain circle was plotted directly on the smith chart using the GaCir tool (for input impedance matching), setting the frequency sweep from $3.5 \mathrm{GHz}$ to $4.5 \mathrm{GHz}$, with a step of $0.5 \mathrm{GHz}$.

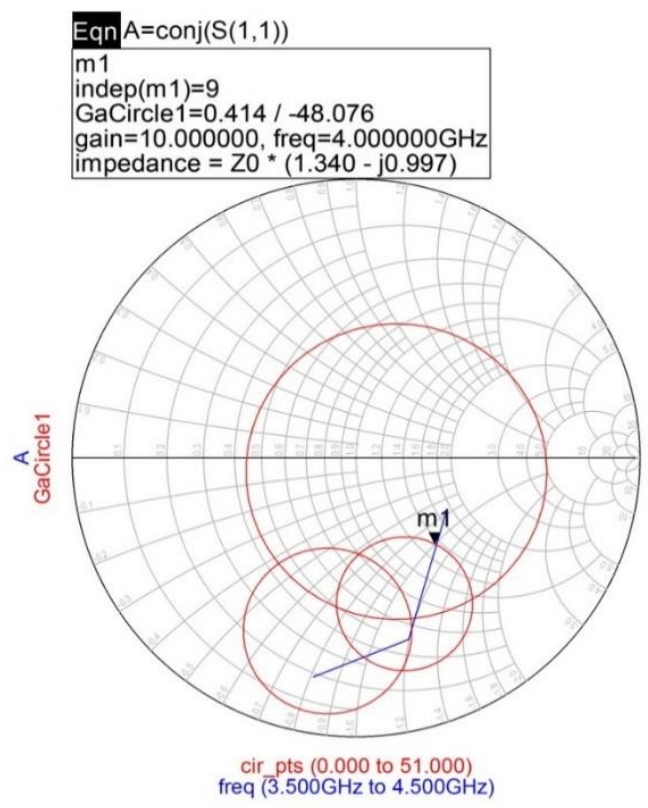

IJEEI, Vol.9, No. 1, March 2021: 210 - 219 
Figure 7. Gain Circle for Input Matching of the Broadband Amplifier.

From the constant gain circle (shown in Figure 7), the normalised impedance of 1.340-j0.99 was chosen as the SmZ1, since it is closest to the $50 \Omega$ point (midpoint). The matching was performed using a smith chart, setting the $\mathrm{SmZ1}=1.340-\mathrm{j} 0.99$, and leaving the SmZ2 unchanged. After matching, new input matching circuit parameters were obtained and presented in Table 4.

Table 4. New Input and output matching circuit parameters for the Broadband Amplifier.

\begin{tabular}{lrrrrr} 
& \multicolumn{2}{c}{ Quarter-wave Transformer } & \multicolumn{3}{c}{ Parallel Open Circuit Stub } \\
\cline { 2 - 6 } & $\mathrm{Z}(\mathrm{Ohm})$ & $\mathrm{E}(\mathrm{Deg})$ & $\mathrm{Z}(\mathrm{Ohm})$ & \multicolumn{2}{c}{$\mathrm{E}(\mathrm{Deg})$} \\
\hline$(\mathrm{SmZ1})$ & 72 & 90 & 50 & 19.948 \\
$(\mathrm{SmZ2})$ & 28 & 90 & 50 & 46.6 \\
\hline
\end{tabular}

These new circuit parameters were employed in the design of the broadband amplifier circuit, and also including microstrip Tees and Steps where necessary. The circuit was simulated and the transducer gain was optimised by tuning, using the tune facility in the ADS. After the gain has been increased, at particular values of the electrical length and impedance, the equivalent values for the length and width of the microstrips are gotten using the Linecalc tool in the ADS. Using these new equivalent values of the line length and width of the microstrip, the Microstrip Broadband Maximum Transducer Power Gain Amplifier was designed as shown in Figure 8. The circuit was simulated, and the maximum gain was plotted. The circuit was tuned at the input to give a flat gain characteristic of about $10 \mathrm{~dB}$, across a frequency of 3.5 to $4.5 \mathrm{GHz}$. The new circuit parameters were recorded in Table 5.

Table 5. Equivalent Line Length and Width of the Microstrip Broadband Amplifier Before and After Tuning.

\begin{tabular}{lll}
\hline Before Tuning & MLIN $($ SmZ1) & MLOC $(\mathbf{S m Z 2})$ \\
\hline Width & $33.984 \mathrm{mil}$ & $61.474 \mathrm{mil}$ \\
Length & $546 \mathrm{mil}$ & $119.145 \mathrm{mil}$ \\
\hline & & \\
& & \\
\hline After Tuning & MLIN (SmZ1) & MLOC $($ SmZ2) \\
\hline Width & $46.42 \mathrm{mil}$ & $61.474 \mathrm{mil}$ \\
Length & $542.137 \mathrm{mil}$ & $214.459 \mathrm{mil}$ \\
\hline
\end{tabular}

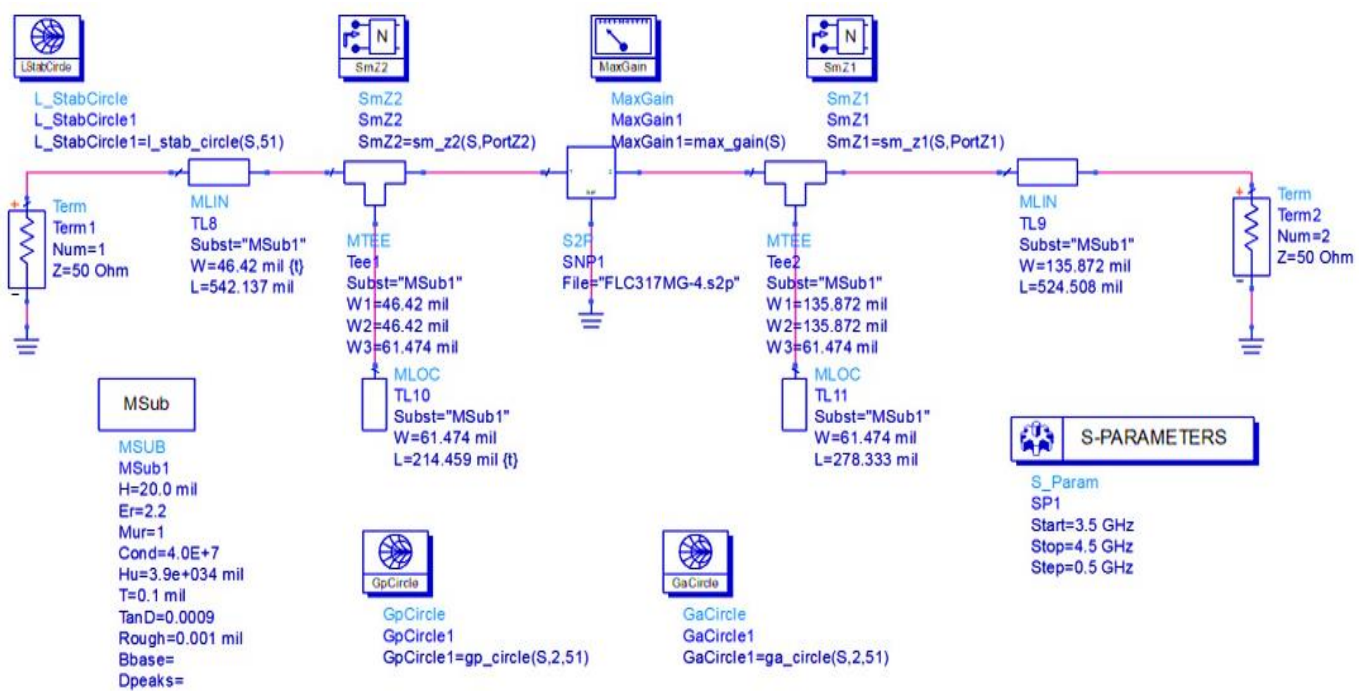

Figure 8. Circuit Schematic of the Microstrip Broadband Amplifier.

\subsubsection{Results}


The microstrip broadband amplifier circuit in Figure 8 was simulated, and the maximum flat gain response and reflection coeffcient obtained is shown in Figure 9.

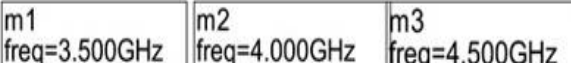
$\mathrm{dB}(\mathrm{S}(2,1))=9.801 \mathrm{~dB}(\mathrm{~S}(2,1))=10.118 \mathrm{~dB}(\mathrm{~S}(2.1))=9.845$
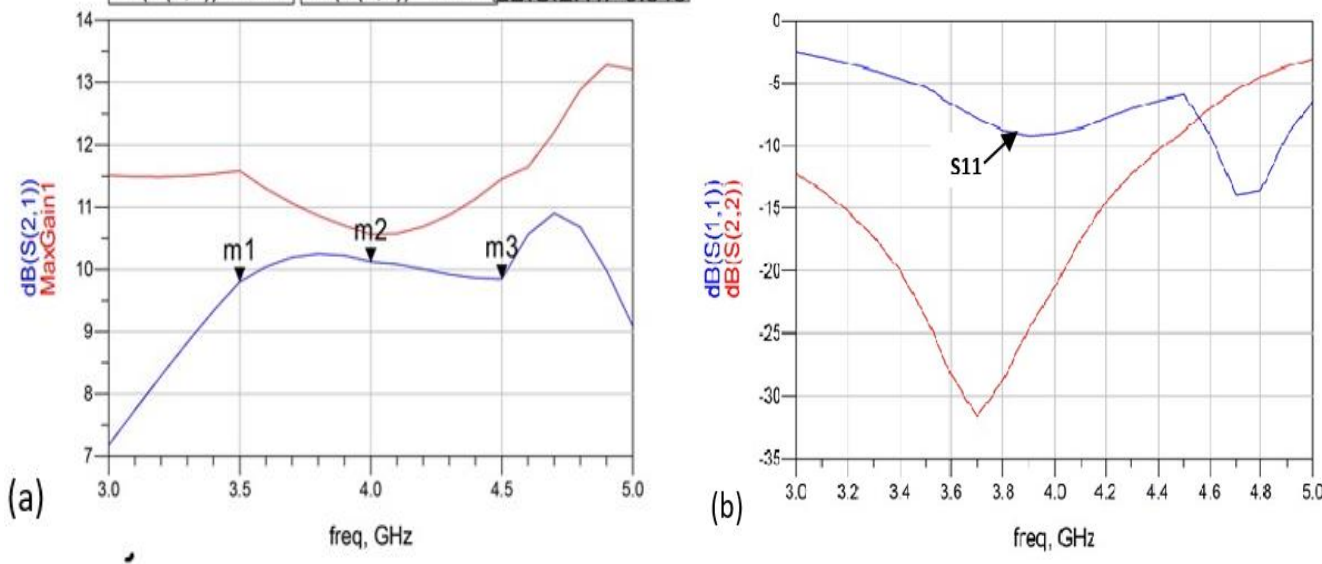

Figure 9.(a) The Gain $S_{21}$ and (b) Reflection Coeffcient $S_{11}$ of the Microstrip Broadband Amplifier.

\subsubsection{Comments}

It was observed that the flat gain broadband was achieved between 3.5 to $4.5 \mathrm{GHz}$. The maximum transducer gain specification of $10 \mathrm{~dB}+/-0.2$ was also achieved.

It was also observed that due to a wider bandwidth, the gain achieved was smaller. This is as a result of the trade-of between the bandwidth and the gain. That is for a broader bandwidth, the gain has to be reduced. In other words, you cannot have more of the bandwidth without having to give up some of the gain. The bandwidth is inversely proportional to the gain.

An increase in the reflection coefficient $\left(S_{11}\right)$ of about $-9 \mathrm{~dB}$ was also observed in the microstrip broadband amplifier. This could be as a result of the losses in real practical conditions of the microstrips, unlike the ideal line components.

\subsubsection{Comparison with Existing Designs}

Various broadband microwave amplifiers with different topologies and frequency band have been compared. Table 6 shows a summary of the different amplifiers and their performance in comparison with this design. It is observed that this design has a_good performance with a high gain at the given frequency band.

Table 6. Comparison of Different Amplifiers and Their Performance

\begin{tabular}{clccc}
\hline Ref & Technology & B.W $(\mathbf{G H z})$ & MaxS21 (dB) & S11 (dB) \\
\hline$[20]$ & $0.18 \mu \mathrm{m} \mathrm{CMOS}$ & $0.9-3.5$ & 8.5 & -3 \\
{$[21]$} & CMOS & $1.65-2.00$ & $5.1 \pm 5$ & -21 \\
{$[22]$} & CMOS & $1.70-3.10$ & 9.8 & -7 \\
{$[23]$} & GaAs PHEMT & 2.45 & 7.51 & -7.497 \\
This Work & FLC317MG-4 FET & $3.5-4.5$ & $9.8-10.118$ & -9 \\
\hline
\end{tabular}

\section{CONCLUSION}

In this paper, a microstrip broadband microwave amplifier was designed and analysed using Agilent ADS. The design procedures and parameters were presented. In the amplifier design process, the S-parameters, stability factor and stability measure were suitable for investigating the stability of the amplifier, and other properties like the gain and losses.

The microstrip broadband amplifier was achieved within a bandwidth of 3.5 to $4.5 \mathrm{GHz}$, and a maximum flat gain of around 9.8 to $10.118 \mathrm{~dB}$. The transducer gain was maximized and the reflection coefficient minimized by matching of the input and output circuits using the smith chart and by tuning (using the tune function) of the ADS. There is a trade-off between the bandwidth and gain of the amplifier. As one increases, the other decreases.

\section{ACKNOWLEDGEMENT}


This research was partly funded by Covenant University, Ota, Ogun State, Nigeria.

\section{REFERENCES}

[1] I. J. Bahl, Fundamentals of RF and Microwave Transistor Amplifiers. New Jersey: John Wiley \& Sons, Inc., 2009.

[2] O. Okoyeigbo, et al., "Comparative Study of MIMO-OFDM Channel Estimation in Wireless Systems," International Review on Modelling and Simulations, vol. 11, no. 3, pp. 158-165, 2018.

[3] N. Kumar and A. Grebennikov, Distributed Power Amplifiers for RF and Microwave Communications. London: Artech House, 2015.

[4] A. Eroglu, Introduction to RF Power Amplifier Design and Simulation. CRC Press, Taylor \& Francis Group, 2016.

[5] P. H. Aaen, J. A. Pla, and C. A. Balanis, "Modeling Techniques Suitable for CAD Based Design of Internal Matching Networks of High-Power RF / Microwave Transistors," IEEE Trans. Microw. Theory Tech., vol. 54, no. 7, pp. 30523059, 2006.

[6] K. C. Gupta, "Emerging Trends in Millimeter-Wave CAD," IEEE Trans. Microw. Theory Tech., vol. 46, no. 6, pp. 747-755, 1998.

[7] A. Rachakh, E. A. Larbi, Z. Jamal, A. Errkik, A. Tajmouati, and L. Mohamed, "A novel Design of A Microstrip Microwave Broadband Power Amplifier for DCS,PCS and UMTS Bands," in International Conference on Wireless Technologies, Embedded and Intelligent Systems (WITS), 2017, pp. 1-5.

[8] L. Wang and D. Chen, "Design of Broadband Power Amplifier Based on ADS," pp. 3-5, 2016.

[9] Q. Wang, W. Che, M. Dionigi, F. Mastri, M. Mongiardo, and G. Monti, "Gains Maximization via Impedance Matching Networks for Wireless Power Transfer," Prog. Electromagn. Res., vol. 164, no. 4, pp. 135-153, 2019.

[10] J. F. White, HIGH FREQUENCY TECHNIQUES: An Introduction to RF and Microwave Engineering. New Jersey, 2004.

[11] C. Poole and I. Darwazeh, Microwave amplifier design. Elsevier Ltd., 2016.

[12] S. Pennisi, G. Scotti and A. Trifiletti, "Avoiding the Gain-Bandwidth Trade Off in Feedback Amplifiers," IEEE Trans. CIRCUITS Syst., vol. 58, no. 9, pp. 2108-2113, 2011.

[13] S.J.G. Gift, and B. Maundy, "Improving the Bandwidth Gain-Independence and Accuracy of the Current Feedback Amplifier," IEEE Trans. CIRCUITS Syst., vol. 52, no. 3, pp. 136-139, 2005.

[14] M. Forouzanfar and M. Joodaki, "Systematic design of hybrid high power microwave amplifiers using large gate periphery GaN HEMTs,” AEU - Int. J. Electron. Commun., vol. 84, no. September 2017, pp. 225-233, 2018.

[15] E. da Silva, High Frequency and Microwave Engineering. Butterworth-Heinemann, 2001.

[16] D. K. Misra, RADIO-FREQUENCY AND MICROWAVE COMMUNICATION CIRCUITS, Second. New Jersey: John Wiley \& Sons, Inc, 2004.

[17] D. M. Pozar, Microwave Engineering, 4th ed. John Wiley \& Sons, Inc., 2012.

[18] B. Abdollahi, B. Mesgari, S. Saeedi, and A. Nabavi, "Stability analysis and compensation technique for lowvoltage regulated cascode transimpedance amplifier," Microelectronics J., vol. 71, no. August 2016, pp. 37-46, 2018.

[19] J. M. Rollett, "Stability and Power-Gain Invariants of Linear Twoports *," IRE Trans. Circuit Theory, vol. 9, no. 1, pp. 29-32, 1962.

[20] R. Sapawi, R. K. Pokharel, D. A. A. Mat, H. Kanaya, and K. Yoshida, "A 0.9-3.5 GHz High Linearity, Good Efficiency CMOS Broadband Power Amplifier Using Stagger Tuning Technique," Microw. Opt. Technol. Lett. vol. 54, no. 12, pp. 2881-2884. 2012.

[21] H. Aniktar, H. Sjoland, J. H. Mikkelsen, and T. Larsen, "A class-AB 1.65GHz-2GHz broadband CMOS medium power amplifier," NORCHIP, 2005. pp. 269-272, 2005.

[22] D. Kalim, D. Erguvan, and R. Negra, "A $1.7 \mathrm{GHz}-$ to-3.1 GHz fully integrated broadband class-E power amplifier in $90 \mathrm{~nm}$ CMOS" 6th Conference on Ph.D. Research in Microelectronics \& Electronics. Berlin. pp. 1-4, 2010.

[23] A. Rasmi, A. Marzuki, M. R. C. Rose, M. AzmiI, and A. I. A. Rahim, “A 2.4 GHz packaged power amplifier using GaAs PHEMT technology,” IEEE Regional Symposium on Micro and Nano Electronics. Kota Kinabalu, pp. 148$151,2011$. 